\title{
Optimal Placement and Sizing of Fault Current Limiters in Distributed Generation Systems Using a Hybrid Genetic Algorithm
}

\author{
Navid Bayati \\ Department of Electrical Engineering \\ Amirkabir University of Technology \\ Tehran, Iran \\ n-bayati@aut.ac.ir
}

\author{
Seyed Hossein H. Sadeghi \\ Department of Electrical Engineering \\ Amirkabir University of Technology \\ Tehran, Iran \\ sadeghi@aut.ac.ir
}

\author{
Amir Hosseini \\ Department of Electrical Engineering \\ Amirkabir University of Technology \\ Tehran, Iran \\ hosseini.amir@aut.ac.ir
}

\begin{abstract}
Distributed Generation (DG) connection in a power system tends to increase the short circuit level in the entire system which, in turn, could eliminate the protection coordination between the existing relays. Fault Current Limiters (FCLs) are often used to reduce the short-circuit level of the network to a desirable level, provided that they are dully placed and appropriately sized. In this paper, a method is proposed for optimal placement of FCLs and optimal determination of their impedance values by which the relay operation time, the number and size of the FCL are minimized while maintaining the relay coordination before and after DG connection. The proposed method adopts the removal of low-impact FCLs and uses a hybrid Genetic Algorithm (GA) optimization scheme to determine the optimal placement of FCLs and the values of their impedances. The suitability of the proposed method is demonstrated by examining the results of relay coordination in a typical DG network before and after DG connection.
\end{abstract}

Keywords- hybrid GA; FCL; placement; DG; relay

\section{INTRODUCTION}

In power systems, the short-circuit current will be examined by a Directional Over-Current Relay (DOCR) and, if necessary, break command be given to a Circuit Breaker (CB). Each DOCR defines a certain breaking time based on the shortcircuit current which passes through it. If this performance fails, the backup DOCR will be activated after a minimum time interval. The coordination among DOCRs is very important in power system protection. Coordination between DOCRs is usually adjusted using pickup current $\left(I_{p}\right)$ and the Time Multiplier Setting (TMS). The adjustment of TMS in DOCR is a linear problem and the adjustment of $I_{p}$ is a non-linear problem. For the optimal adjustment of coordination between DOCRs, the optimal adjustment of TMS and $I_{p}$ in [1-2] has been performed using GA and particle swarm optimization (PSO) methods. In [3], relay adjustment is done by changing the network's topology.

Power network's structure and short-circuit level in power systems change when connecting the Distributed Generation (DGs). In fact, after connecting DG, the increase in short circuit level causes a change in the coordination of DOCRs and may result in a protection miscoordination. There are several ways to reduce the short circuit level in the presence of DG. In [4] it has been stated that when there is a fault in the short circuit connection, DG connection should be interrupted; this method would cut off access to the DG after handling the fault which creates operational problems.

One of the best ways to reduce the short circuit level is to use Fault Current Limiters (FCLs), which reduce the shortcircuit current with an increased impedance during faults. Although using FCL is very costly, it offers many advantages such as reducing short-circuit current, reducing the voltage sag during fault and increasing the stability and reliability of the network. Since FCL connection is costly, proper placement of the device and reducing the number of FCLs are very important. In [5], the sensitivity factor method is used to determine the optimal placement of FCLs. In [6], GAs are used for optimal FCL placement. In addition, in order to reduce costs, optimum values of FCL's impedances should be considered. In [7-8] various methods have been used to optimize the amount of FCLs. In this article, Hybrid GA is implemented which uses a combination of GA and Linear Programming (LP) to optimize the amount of FCL. Also, a new method is presented for proper placement of FCLs that minimizes the number of FCLs. Here, the aim of placing the FCL is to restore the coordination of DOCR in the presence of DGs which reduces the operating time of DOCRs as well. The advantages of this method have been assessed in a network under several scenarios. Finally the proposed method is compared with conventional algorithms such as GAs.

\section{RELAY COORDINATION}

The aim of coordination of DOCRs is to adjust the TMS and Ip for each DOCR in a way that the time difference between the main DOCR and a backup DOCR of a specific Coordination Time Interval (CTI) is more. Moreover, this means that if the main DOCR is not operated, the backup DOCR will be operated after CTI. If the time difference of the operation between the main relay and the backup relay is less than CTI, miscoordination will occur. Also the main relay's 
operating time should be minimized. In other words, the optimization of the objective function is defined as follows [9]:

$$
O F=\sum_{i=1}^{N} t_{i}
$$

In the above equation, $N$ is the number of relays and $t_{i}$ is the operating time of the main relay. According to IEC standard, this time performance is defined as follows:

$$
t_{i}=\frac{0.14}{\left(\frac{I_{s c_{i}}}{I_{p_{i}}}\right)^{0.02}-1} T M S_{i}
$$

In the above equation, TMSi and Ipi are the settings of ith relay and $I_{s c i}$ is the short-circuit current passing through the main relay.

In the optimization of DOCR's coordination, a problem is stated where its constraints are as follows:

$$
t_{j}-t_{i} \geq C T I
$$

In the above equation, $t_{j}$ is the operating time of the backup relay and $t_{i}$ is the main relay operating time. CTI is usually considered as 0.3 second.

$$
T M S_{\min } \leq T M S_{i} \leq T M S_{\max }
$$

A maximum and a minimum value are defined for TMS and the value of TMS for Each relay should be considered in this range.

$$
I_{P_{\min }} \leq I_{P} \leq I_{P_{\max }}
$$

In the above equation, $I_{P \min }$ must be more than the maximum load current and $I_{P \max }$ should be less than shortcircuit current. Using the constraints mentioned in (2) to (5) and using the fitness function which was defined in (1), we can adjust the DOCRs with the help of optimization algorithms such as GA.

\section{OPTIMIZING FCL IMPEDANCE}

The purpose of this article is to optimize the impedance value of FCL in a way that it restores the coordination of DOCRs, and optimizes their performance time; also due to economic reasons, the impedance value of FCLs should be considered optimally. In this article, a resistive type FCL is used because its cost is lower compared to other types of FCL. The fitness function used in this paper is applied to determine the importance of the optimizing the weighting. The fitness function is composed of two parts, part one is related to the reducing time of the DOCRs' performance and part two is related to optimization of the FCL's resistance.

Fitness function is as follows:

$$
F=\sum_{i=1}^{N} t_{i}+\omega \sum_{j=1}^{L} R_{i}
$$

In the above equation, $\sum_{i=1}^{N} t_{i}$ represents the sum of the operating time of the main relay, $\sum_{j=1}^{L} R_{i}$ indicates the total FCL's resistance used in the network, and $\omega$ is the weight factor for the resistance. In this article, $\omega$ is considered as 10 .

Also the optimization constraints are as follows:

$$
\begin{aligned}
& R_{\min } \leq R \leq R_{\max } \\
& t_{j}-t_{i} \geq C T I
\end{aligned}
$$

In the above equation, $\mathrm{R}$ represents the resistance value of FCL that should be considered between the minimum and maximum values. Also the second constraint represents the difference between the operating time of the backup and the primary relays which should be more than CTI. Because of the constraints, we can consider a penalty factor for the fitness function. By considering the penalty factor, the objective function is defined as follows[5]:

$$
F=\sum_{i=1}^{N} t_{i}+\omega \sum_{j=1}^{L} R_{i}+\sum_{i=1}^{N} K_{1}+\sum_{j=1}^{L} K_{2}
$$

In the above equation, $\mathrm{K} 1$ and $\mathrm{K} 2$ are considered as penalty factors. If the constraint which is shown in (7-a) is applied, the amount of $\mathrm{K} 1$ will be equal to zero and if the constraint is not applied, the amount of K1 will be equal to 500. The same consideration is applied for 7-b.

\section{OPTIMAL FCL PLACEMENT}

For optimum FCL utilization in power systems, restoration of protective miscoordination of DOCR caused by DG and the reducing the cost of FCL installations, proposed method must first determine and optimize the number and placement of FCLs and then determine the amount of each FCL. For this purpose, at this stage, a method is proposed for the optimal placement of FCL. After the installation of DG in the power systems, the protection coordination of DOCR is eliminated due to changes of short-circuit level. Again, it is necessary to use FCL to restore protection coordination. But the increase in the number of FCLs and installation of additional FCLs is very expensive. For this reason, it is necessary to optimize the number of FCLs. For this purpose, after the optimization of FCLs by optimization algorithms, the value of the FCL resistance is determined. First, it is assumed that all lines are candidates for FCL placement. Then, after optimizing and determining the resistance of the FCLs, the candidate locations having the least effects on eliminating miscoordination would be specified - FCLs at these places have the least resistance- So the places whose resistance is less than a certain amount can be determined and removed from the candidates. Then the optimization can be applied again. The difference is that the optimization of the second low-impact places has been removed from the FCL candidates' site. In this method, the number of FCLs is reduced as much as possible and thus the cost is reduced. 


\section{HYBRID GA}

Optimization problems are defined both in linear and nonlinear parts. The linear problems can be optimized by LP algorithms and the nonlinear problems can be optimized with evolutionary algorithms such as the GA. In hybrid GA which has arisen from the combination of GA and LP, the linear parts of optimization problem are optimized by LP method and the non-linear parts of optimization problem are optimized by GA. [3] The advantages of this method are reducing simulation time and achieving better fitness function. Also, this method improves optimized solutions due to reduction of GA variables. In this paper, hybrid GA is used for optimizing coordination between DOCRs and optimizing the resistance value of FCLs. In the problem of coordination of DOCRs, the linear part determines the TMS and the non-linear parts determine $I_{p}$. In the optimization problem of FCL's resistance, the linear parts determine the optimization of the FCL's resistance and the non-linear parts determine the optimization of the time performance of the relays. Hybrid GA flowchart is used to optimize FCL's resistance which is shown in Figure 1.

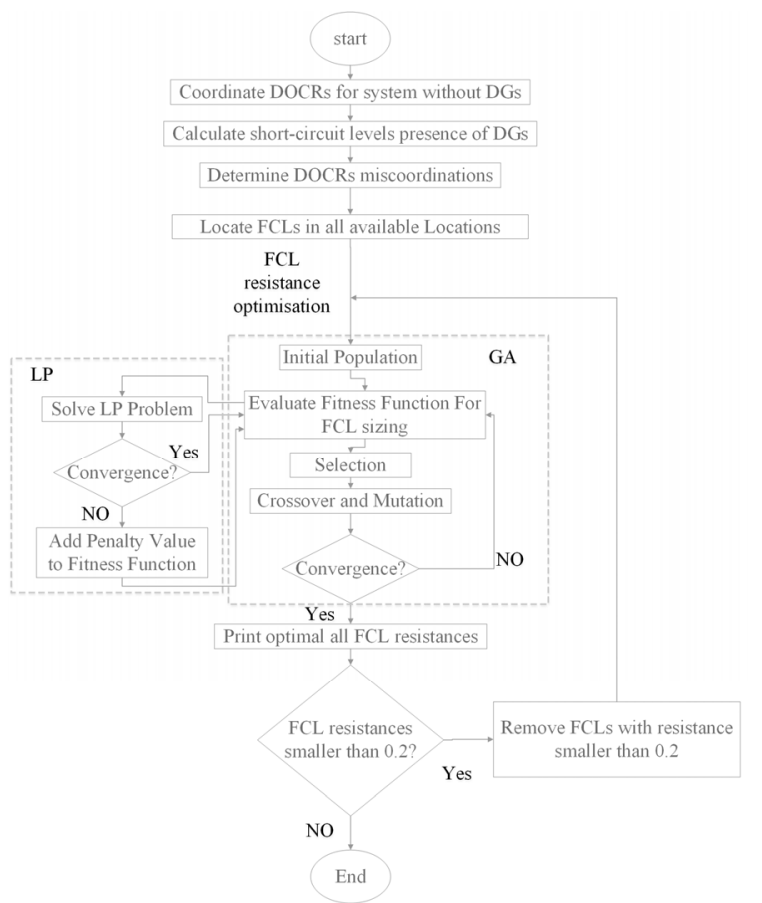

Fig. 1. Hybrid GA for optimize FCL resistance

GA is composed of multiple parts: Initialization, crossover, evaluation and mutation. These are further explained below:

- Initialization: at this stage, the need to optimize the initial population is conducted randomly and this initial population is used to build the next generation by evaluation.

- Evaluation: in GA, the value of each generation or string is determined by the fitness function and it becomes clear at this stage which parts of the population are to be used in the next step. In this paper, the used fitness function has been shown in (8).
- Crossover: This stage is usually probabilistic, the population which has a more desirable fitness function is more likely to be selected to participate in the next generations.

- Mutation: At this stage, a new population is created to participate in the GA which was not present in the previous population. This step makes it possible to increase the search in all cases of the problem.

In the hybrid GA, the LP is described as a sub-problem being applied to the fitness function, in other words, the LP first specifies the linear values and then these values are given to GA and the GA optimizes the values of non-linear problem.

\section{SIMULATION AND RESULTS}

The proposed method is applied on an 8-bus system with 14 DOCRs which is shown in Figure 2 [10]. The DG has a capacity of 50MVA, which is connected to bus 2 in the first scenario and they are connected to the buses 2 and 5 in the second scenario. The optimization method is conducted in a way that it first optimizes the coordination between DOCR regardless of DG. At this stage, (1) is the fitness function of the DOCR coordination. After DOCR's coordination, with the addition of DG to the network, the short-circuit levels of the power network are changed and this change may cause protection miscoordination. Then, in the next stage, by inserting the FCLs to the network and measuring the shortcircuit currents and investigating the protection coordination, optimization is performed using hybrid GA, equation 7 and 8 have considered the fitness function and constraints, respectively.

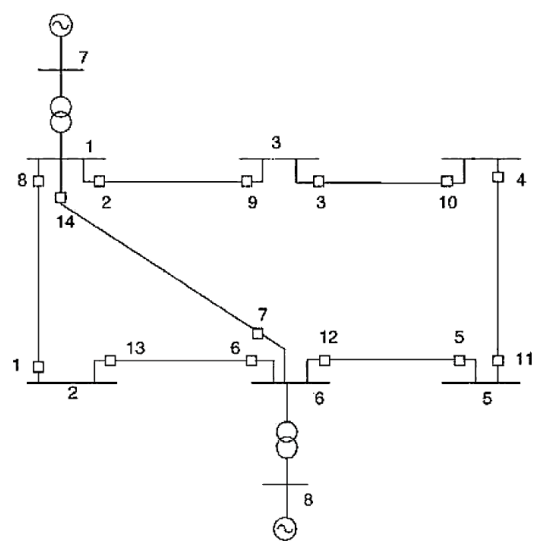

Fig. 2. 8-bus system

Table I shows the DOCR settings without the presence of DG. To have a better protection coordination the following constraint is used; if the value of the constraint is positive, the protection coordination will be established:

$$
\text { constraint }=t_{\text {backup }}-t_{\text {primary }}-C T I
$$

In the first scenario, DG has only been connected to bus (2). As it is shown in Table II, a number of miscoordinations has arisen in DOCRs when DG is connected to bus 2. In other 
words, the difference between the main and the backup relay's operation time is less than the CTI. For this reason, the FCL has been used to restore the protection coordination and its resistance value has been determined using the proposed method. As indicated in Table II, 15 pairs of DOCRs have been miscoordinated after installing the DG. As it has been shown in Table II at the first stage, all lines are candidates for the placement of FCLs. After determining the FCL's resistance by hybrid GA, all FCLs with a resistance less than $0.2 \mathrm{pu}$ which is determined considering economic issues, are regarded as low impact FCLs. So these FCLs are removed and optimization is done again. As indicated in Table IV, by removing the specified FCLs, the protection coordination of DOCRs is restored and consequently, the costs of installing FCLs are reduced.

TABLE I. DOCR SETTINGS WITHOUT THE PRESENCE OF DG

\begin{tabular}{|c|c|c|}
\hline Relay no. & $\boldsymbol{I}_{\boldsymbol{p}}$ & TMS \\
\hline 1 & 596 & 0.058 \\
\hline 2 & 120 & 0.573 \\
\hline 3 & 338 & 0.297 \\
\hline 4 & 123 & 0.332 \\
\hline 5 & 120 & 0.228 \\
\hline 6 & 120 & 0.262 \\
\hline 7 & 80 & 0.571 \\
\hline 8 & 120 & 0.488 \\
\hline 9 & 353 & 0.050 \\
\hline 10 & 120 & 0.198 \\
\hline 11 & 120 & 0.330 \\
\hline 12 & 120 & 0.482 \\
\hline 13 & 120 & 0.354 \\
\hline 14 & 80 & 0.501 \\
\hline
\end{tabular}

TABLE II. RELAY PAIRS CONSTRAINTS

\begin{tabular}{|c|c|c|c|}
\hline $\begin{array}{c}\text { Primary } \\
\text { Relay no. }\end{array}$ & $\begin{array}{c}\text { Backup } \\
\text { Relay no. }\end{array}$ & $\begin{array}{c}\text { Constraint } \\
\text { (without DG) }\end{array}$ & $\begin{array}{c}\text { Constraint } \\
\text { (with DG) }\end{array}$ \\
\hline 1 & 6 & 0.00 & 0.086 \\
\hline 2 & 1 & 0.00 & -0.919 \\
\hline 2 & 7 & 0.00 & -0.052 \\
\hline 3 & 2 & 0.00 & -0.017 \\
\hline 4 & 3 & 0.00 & -0.058 \\
\hline 5 & 4 & 0.00 & -0.024 \\
\hline 6 & 5 & 0.49 & 0.272 \\
\hline 6 & 14 & 0.37 & 0.291 \\
\hline 7 & 5 & 0.00 & -0.164 \\
\hline 7 & 13 & 0.00 & -0.362 \\
\hline 8 & 7 & 0.12 & 0.035 \\
\hline 8 & 9 & 0.91 & -0.407 \\
\hline 9 & 10 & 0.00 & -0.012 \\
\hline 10 & 11 & 0.00 & -0.019 \\
\hline 11 & 12 & 0.00 & -0.017 \\
\hline 12 & 13 & 0.11 & -0.256 \\
\hline 12 & 14 & 0.00 & -0.038 \\
\hline 13 & 8 & 0.00 & 0.144 \\
\hline 14 & 1 & 0.14 & -0.768 \\
\hline 14 & 9 & 0.95 & -0.343 \\
\hline
\end{tabular}

Also as it is shown in Table IV, all protection coordination of DOCRs are restored after the installing the FCLs. Also optimization is performed using hybrid GA and GA. In hybrid GA, the value of the fitness function is less than that of GA and this reflects the strength of the proposed method. In the second scenario, DGs are connected to buses number 2 and 5. As indicated in Table V, 15 pairs of DOCR s have miscoordinated after installing two DGs. After installing the FCLs on the lines and the optimizing the values of the FCLs, as shown in Table VI, all coordination of DOCRs are restored. The amount and the location of the FCL have been shown in Table VII.

TABLE III. FCL RESISTANCE AND PLACEMENT

\begin{tabular}{|c|c|c|c|}
\hline $\begin{array}{c}\text { Line } \\
\text { no. }\end{array}$ & $\begin{array}{c}\text { Resistance } \\
\text { (first stage) }\end{array}$ & $\begin{array}{c}\text { Resistance ( second stage } \\
\text { using hybrid GA) }\end{array}$ & $\begin{array}{c}\text { Resistance ( second } \\
\text { stage using GA) }\end{array}$ \\
\hline 1 & 0.6699 & 0.6553 & 0.7281 \\
\hline 2 & 0.4066 & 0.3666 & 0.3496 \\
\hline 3 & 0.0465 & 0.0000 & 0.0000 \\
\hline 4 & 0.1924 & 0.0000 & 0.0000 \\
\hline 5 & 0.2539 & 0.4883 & 0.8321 \\
\hline 6 & 0.6994 & 0.7675 & 0.7087 \\
\hline 7 & 0.3164 & 0.2057 & 0.8326 \\
\hline
\end{tabular}

TABLE IV. RELAY PAIRS CONSTRAINTS WITH FCL

\begin{tabular}{|c|c|c|c|}
\hline $\begin{array}{l}\text { Primary } \\
\text { Relay no. }\end{array}$ & $\begin{array}{c}\text { Backup } \\
\text { Relay no. }\end{array}$ & $\begin{array}{c}\text { Constraint } \\
\text { (hybrid GA) }\end{array}$ & $\begin{array}{c}\text { Constraint } \\
\text { (GA) }\end{array}$ \\
\hline 1 & 6 & 0.4125 & 0.3778 \\
\hline 2 & 1 & 0.0181 & 0.4470 \\
\hline 2 & 7 & 0.0190 & 0.4649 \\
\hline 3 & 2 & 0.0206 & 0.0346 \\
\hline 4 & 3 & 0.2731 & 0.2644 \\
\hline 5 & 4 & 0.0143 & 0.0120 \\
\hline 6 & 5 & 0.4837 & 0.3814 \\
\hline 6 & 14 & 0.3246 & 0.8334 \\
\hline 7 & 5 & 0.0208 & 0.1050 \\
\hline 7 & 13 & 0.0679 & 0.0004 \\
\hline 8 & 7 & 0.0630 & 0.6060 \\
\hline 8 & 9 & 0.9746 & 0.3135 \\
\hline 9 & 10 & 0.0088 & 0.0638 \\
\hline 10 & 11 & 0.1250 & 0.2287 \\
\hline 11 & 12 & 0.1804 & 0.2889 \\
\hline 12 & 13 & 0.2028 & 0.0686 \\
\hline 12 & 14 & 0.0249 & 0.4151 \\
\hline 13 & 8 & 0.6341 & 0.7164 \\
\hline 14 & 1 & 0.1728 & 0.6842 \\
\hline \multirow[t]{2}{*}{14} & 9 & 0.9973 & 0.4096 \\
\hline & $\begin{array}{c}\text { Fitness } \\
\text { function }\end{array}$ & 40.4 & 52.1 \\
\hline
\end{tabular}

\section{CONCLUSION}

This paper proposes an efficient method to determine the optimal resistance values and optimal placement of Fault Current Limiters (FCLs) in a Distributed Generation (DG) network to ensure protection coordination of Directional OverCurrent Relays (DOCRs) both before and after DG connection. The proposed method adopts the removal of lowimpact FCLs and uses a hybrid Genetic Algorithm (GA) optimization scheme to determine the optimal placement of FCLs and the values of their resistances. The implementation of the proposed method on a typical DG network has shown that that the existence of FCLs reduces the operating times of DOCRs while restoring their protection coordination in the presence of DG. It has also been shown that the proposed method minimizes the required number of FCLs, thus reducing the installation costs. 
TABLE V. RELAY PAIRS CONSTRAINTS WITH 2 DGS

\begin{tabular}{|c|c|c|c|}
\hline $\begin{array}{c}\text { Primary } \\
\text { Relay no. }\end{array}$ & $\begin{array}{c}\text { Backup } \\
\text { Relay no. }\end{array}$ & $\begin{array}{c}\text { Constraint } \\
\text { (without DG) }\end{array}$ & $\begin{array}{c}\text { Constraint } \\
\text { (with DG) }\end{array}$ \\
\hline 1 & 6 & 0.00 & 0.0287 \\
\hline 2 & 1 & 0.00 & -0.9331 \\
\hline 2 & 7 & 0.00 & -0.1457 \\
\hline 3 & 2 & 0.00 & -0.0168 \\
\hline 4 & 3 & 0.00 & -0.2068 \\
\hline 5 & 4 & 0.00 & 0.0000 \\
\hline 6 & 5 & 0.49 & -0.2336 \\
\hline 6 & 14 & 0.37 & 0.2143 \\
\hline 7 & 5 & 0.00 & -0.6487 \\
\hline 7 & 13 & 0.00 & -0.3608 \\
\hline 8 & 7 & 0.12 & -0.0438 \\
\hline 8 & 9 & 0.91 & -0.9382 \\
\hline 9 & 10 & 0.00 & -0.0359 \\
\hline 10 & 11 & 0.00 & -0.0586 \\
\hline 11 & 12 & 0.00 & 0.0235 \\
\hline 12 & 13 & 0.11 & -0.2987 \\
\hline 12 & 14 & 0.00 & -0.1387 \\
\hline 13 & 8 & 0.00 & 0.0281 \\
\hline 14 & 1 & 0.14 & -0.7835 \\
\hline 14 & 9 & 0.95 & -0.8905 \\
\hline
\end{tabular}

TABLE VI. RELAY PAIRS CONSTRAINTS WITH 2 DGS AND FCL

\begin{tabular}{|c|c|c|c|}
\hline $\begin{array}{c}\text { Primary } \\
\text { Relay no. }\end{array}$ & $\begin{array}{c}\text { Backup } \\
\text { Relay no. }\end{array}$ & $\begin{array}{c}\text { Constraint } \\
\text { (hybrid GA) }\end{array}$ & $\begin{array}{c}\text { Constraint } \\
\text { (GA) }\end{array}$ \\
\hline 1 & 6 & 0.4175 & 1.0427 \\
\hline 2 & 1 & 0.0030 & 0.0085 \\
\hline 2 & 7 & 0.2244 & 0.4860 \\
\hline 3 & 2 & 0.0679 & 0.0153 \\
\hline 4 & 3 & 0.2554 & 0.1536 \\
\hline 5 & 4 & 1.0404 & 0.9189 \\
\hline 6 & 5 & 0.6701 & 0.5322 \\
\hline 6 & 14 & 0.6146 & 0.8668 \\
\hline 7 & 5 & 0.1591 & 0.0058 \\
\hline 7 & 13 & 0.0317 & 0.8896 \\
\hline 8 & 7 & 0.3596 & 0.6248 \\
\hline 8 & 9 & 0.5350 & 0.0000 \\
\hline 9 & 10 & 0.0910 & 0.0084 \\
\hline 10 & 11 & 0.2849 & 0.2604 \\
\hline 11 & 12 & 2.1610 & 1.8342 \\
\hline 12 & 13 & 0.1270 & 0.9622 \\
\hline 12 & 14 & 0.1990 & 0.4129 \\
\hline 13 & 8 & 0.6621 & 0.6842 \\
\hline 14 & 1 & 0.2204 & 0.2512 \\
\hline 14 & 9 & 0.6172 & 0.1040 \\
\hline & $\begin{array}{c}\text { Fitness } \\
\text { function }\end{array}$ & 71.25 & 81.00 \\
\hline
\end{tabular}

TABLE VII. FCL RESISTANCE AND PLACEMENT

\begin{tabular}{|c|c|c|c|}
\hline $\begin{array}{c}\text { Line } \\
\text { no. }\end{array}$ & $\begin{array}{c}\text { Resistance } \\
\text { (first stage) }\end{array}$ & $\begin{array}{c}\text { Resistance ( second } \\
\text { stage using hybrid GA) }\end{array}$ & $\begin{array}{c}\text { Resistance ( second } \\
\text { stage using GA) }\end{array}$ \\
\hline 1 & 0.6800 & 0.6625 & 0.6636 \\
\hline 2 & 0.1687 & 0.3350 & 0.2568 \\
\hline 3 & 0.7600 & 0.0000 & 0.0000 \\
\hline 4 & 0.2400 & 0.9783 & 0.9416 \\
\hline 5 & 1.2600 & 1.6860 & 1.5008 \\
\hline 6 & 0.9600 & 0.7635 & 1.6103 \\
\hline 7 & 0.3600 & 0.6314 & 0.9259 \\
\hline
\end{tabular}

\section{REFERENCES}

[1] F. Razavi, H. A. Abyaneh, M. Al-Dabbagh, R. Mohammadi, H. Torkaman, "A new comprehensive genetic algorithm method for optimal overcurrent relays coordination", Electric Power Systems Research, Vol. 78, No. 4, pp. 713-720, 2008

[2] J. B. Ekanayake, "A systematic approach for phase-fault over-current protection coordination of complex networks", International Journal of Electrical Engineering Education, Vol. 45, No. 3, pp. 229-238, 2008

[3] A. S. Noghabi, H. R. Mashhadi, J. Sadeh, "Optimal coordination of directional overcurrent relays considering different network topologies using interval linear programming", IEEE Transactions on Power Delivery, Vol. 25, No. 3, pp. 1348-1354, 2010

[4] W. K. A. Najy, H. H. Zeineldin, W. L. Woon, "Optimal protection coordination for microgrids with grid-connected and islanded capability", IEEE Transactions on Industrial Electronics, Vol. 60, No. 4, pp. $1668-1677,2013$

[5] J. -H. Teng, C. -N. Lu, "Optimum fault current limiter placement with search space reduction technique", IET Generation, Transmission \& Distribution, Vol. 4, No. 4, pp. 485-494, 2010

[6] S. A. Hosseini, H. A. Abyaneh, S. H. H. Sadeghi, F. Razavi, "Merging the retrieval of the protection coordination of distribution networks equipped with DGs in the process of their siting and sizing", Journal of Renewable and Sustainable Energy, Vol. 8, No. 3, 2016

[7] H. -C. Jo, S. -K. Joo, K. Lee. "Optimal placement of superconducting fault current limiters (SFCLs) for protection of an electric power system with distributed generations (DGs)", IEEE Transactions on Applied Superconductivity, Vol. 23, No. 3, Article Sequence Number: 5600304, 2013

[8] W. El-Khattam, T. S. Sidhu, "Restoration of directional overcurrent relay coordination in distributed generation systems utilizing fault current limiter", IEEE Transactions on Power Delivery, Vol. 23, No. 2, pp. 576-585, 2008

[9] A. S. Noghabi, J. Sadeh, H. R. Mashhadi, "Considering different network topologies in optimal overcurrent relay coordination using a hybrid GA", IEEE Transactions on Power Delivery, Vol. 24, No. 4, pp. 1857-1863, 2009

[10] H. H. Zeineldin, E. F. El-Saadany, M. M. A. Salama, "Optimal coordination of overcurrent relays using a modified particle swarm optimization", Electric Power Systems Research, Vol. 76, No. 11, pp. 988-995, 2006 